\title{
BROYDEN'S METHOD FOR OPERATORS WITH REGULARLY CONTINUOUS DIVIDED DIFFERENCES
}

\author{
Anatoly M. Galperin
}

\begin{abstract}
We present a new convergence analysis of popular Broyden's method in the Banach/Hilbert space setting which is applicable to nonsmooth operators. Moreover, we do not assume a priori solvability of the equation under consideration. Nevertheless, without these simplifying assumptions our convergence theorem implies existence of a solution and superlinear convergence of Broyden's iterations. To demonstrate practical merits of Broyden's method, we use it for numerical solution of three nontrivial infinite-dimensional problems.
\end{abstract}

\section{Introduction}

Trying to overcome disadvantages of Newton's method

$$
x_{+}:=x-\mathbf{f}^{\prime}(x)^{-1} \mathbf{f}(x)
$$

for solving systems of nonlinear equations

$$
\mathbf{f}(x)=0, \mathbf{f}: \mathbb{R}^{n} \supset D \rightarrow \mathbb{R}^{n},
$$

C. Broyden proposed in [2] to replace the inverse Jacobian $\mathbf{f}^{\prime}(x)^{-1}$ in (1.1) by some its approximation (an $n \times n$ matrix) A satisfying the equation

$$
\mathbf{A}\left(\mathbf{f}(x)-\mathbf{f}\left(x_{-}\right)\right)=x-x_{-},
$$

where $x_{-}$denotes the iteration preceding the current one $x$. Since this equation does not define A uniquely, it determines a class of iterative methods that Broyden refers to as quasi-Newton methods. Particular methods of this class differ by the choice of $\mathbf{A}$ among solutions of (1.3). Broyden's choice advocated in $[2]$ is

$$
\mathbf{A}_{+}:=\mathbf{A}-\frac{\mathbf{A f}\left(x_{+}\right)}{\mathbf{f}(x)^{T} \mathbf{A}^{T} \mathbf{A}\left(\mathbf{f}\left(x_{+}\right)-\mathbf{f}(x)\right)} \mathbf{f}(x)^{T} \mathbf{A}^{T} \mathbf{A} .
$$

Received February 2, 2014; Revised August 14, 2014.

2010 Mathematics Subject Classification. 47J05, 47J25, 65J15.

Key words and phrases. nonlinear operator equations, Broyden's method, convergence analysis, regular continuity. 
Broyden's method has proved to be successful in practice for solving (1.2). Being paraphrased for operator equations

$$
\mathbf{f}(x)=0, \mathbf{f}: \mathbb{X} \supset D \rightarrow \mathbb{H}
$$

where $\mathbf{f}$ acts from a subset $D$ of a Banach space $\mathbb{X}$ into a Hilbert one $\mathbb{H}$, it becomes

$$
x_{+}:=x-\mathbf{A f}(x), \mathbf{A}_{+}:=\mathbf{A}-\frac{\mathbf{A f}\left(x_{+}\right)}{\left\langle\mathbf{A}^{*} \mathbf{A} \mathbf{f}(x), \mathbf{f}\left(x_{+}\right)-\mathbf{f}(x)\right\rangle}\left\langle\mathbf{A}^{*} \mathbf{A f}(x), \cdot\right\rangle .
$$

Here $\mathbf{A}^{*}$ stands for the adjoint of $\mathbf{A}$ and $\langle\cdot, \cdot\rangle$ is the inner product in $\mathbb{H}$. Broyden's method has since been applied to infinite-dimensional problems $[13,14,15]$ under various simplifying assumptions. There are also convergence analyses of the method in general Hilbert and Banach spaces [10, 21]. In all these studies, operators under discussion are assumed to be Lipschitz smooth. Similar assumptions are made in [22]. Since the method does not involve evaluation of derivatives of the operator, it can be used for solving equations with nondifferentiable operators. In this case, the above mentioned analyses are unapplicable. Another limiting postulate present (explicitly or implicitly) in existing analyses of Broyden's method is solvability of the equation (1.4). This assumption is often difficult to verify a priori. So, it should be dropped too. Moreover, the focus of the analysis is most often on conditions that ensure superlinear convergence property of Broyden's method, well known from its analyses in finite-dimensional spaces and their extensions to Hilbert ones $[8,16]$. This property is proved also [20], when Lipschitz smoothness of the operator is not assumed.

The purpose of the present paper is to offer a new analysis of Broyden's method in Banach/Hilbert space setting free of unnecessary assumptions restricting applicability of other analyses available in literature. Our analysis is based on Kantorovich's majorization principle, which stems from Kantorovich's investigation of Newton's method in Banach space setting [11, Ch. XVIII]. However, the analysis we are going to present here differs from the others not only in methodology, but in several other aspects too:

- It is applicable to nonsmooth operators, since differentiability of $\mathbf{f}$ is not assumed. Instead, we require that the selected divided difference operator $\left[x_{1}, x_{2} \mid \mathbf{f}\right]$ of $\mathbf{f}$ be regularly continuous $[5,7]$.

- No assumption about the existence of solution is made. On the contrary, our convergence condition, which involves only the starter $\left(x_{0}, \mathbf{A}_{0}\right)$, guarantees its existence.

- We show that if the sequence $\left(x_{n}, \mathbf{A}_{n}\right)$ generated by the method (1.5) converges (to $\left(x_{\infty}, \mathbf{A}_{\infty}\right)$ ), it does to a solution of the system $\mathbf{f}(x)=0 \& \mathbf{A}[x, x \mid \mathbf{f}]=$ $\mathbf{I}$, where $\mathbf{I}$ is the identity operator: $\mathbf{I} x=x, \forall x$. This property of the method is very helpful to those interested in solution's sensitivity to small perturbations in the data. 
- The bound on the error $\left\|x_{\infty}-x_{n}\right\|$ obtained implies superlinear rate of convergence. This bound, however, allows to estimate rate of convergence not only asymptotically, but beginning with initial iterations. Moreover, it suggests a new criterion for comparison of efficiency of various iterative methods reflecting their common goal: locate a solution of the equation (1.4).

- The existence condition, the existence and uniqueness radii are shown to be exact.

- The approach to the analysis of the method (1.5) adopted in this paper provides yet another example (in addition to those considered in [6]) of the role invariants of systems of difference equations can play in convergence analysis of iterative methods.

The use of regularly continuous divided differences makes necessary recalling some definitions and basic facts from [7] needed for understanding subsequent developments. This material makes up the next section. Section 3 contains derivation of the majorant generator and the convergence lemma preparing convergence theorem in Section 4. The short fifth section contains brief discussion of rate of convergence of Broyden's method. Then we test the method numerically on three nontrivial infinite-dimensional (including nonsmooth) problems to demonstrate method's practicability.

\section{Regularly continuous divided differences}

Though Broyden's method (1.5) is defined for any operator $\mathbf{f}$ acting from a convex subset $D$ of a Banach space (say $\mathbb{X}$ ) into a Hilbert one, the concept of divided difference operator (briefly dd) makes sense in Banach spaces. So, recalling its definition we are speaking in this section about Banach spaces.

Definition 2.1. A linear bounded operator $\mathbf{A}$ from $\mathbb{X}$ into $\mathbb{Y}$ is called a divided difference operator, if for any given pair $\left(x_{1}, x_{2}\right)$ of points of $D$ it satisfies the (secant) equation

$$
\mathbf{A}\left(x_{1}-x_{2}\right)=\mathbf{f}\left(x_{1}\right)-\mathbf{f}\left(x_{2}\right) .
$$

To emphasize its dependence on $x_{1}, x_{2}$, and $\mathbf{f}$, such an operator is denoted by the symbol $\left[x_{1}, x_{2} \mid \mathbf{f}\right]$.

For given $x \in \mathbb{X}$ and $y \in \mathbb{Y}$, linear operators satisfying the equation $\mathbf{A} x=y$ constitute an affine manifold in the space $\mathcal{L}(\mathbb{X}, \mathbb{Y})$ of all bounded linear operators between $\mathbb{X}$ and $\mathbb{Y}$ :

$$
\mathbf{A}_{0} x=y \quad \& \quad \mathbf{A} x=y \Longrightarrow\left(\mathbf{A}-\mathbf{A}_{0}\right) x=0 \Longrightarrow \mathbf{A} \in \mathbf{A}_{0}+\mathcal{L}_{x},
$$

where $\mathcal{L}_{x} \subset \mathcal{L}(\mathbb{X}, \mathbb{Y})$ is the subspace of operators vanishing on $x$. So, the symbol $\left[x_{1}, x_{2} \mid \mathbf{f}\right]$ should be understood as the notation for this manifold or, more precisely, as its particular representative selected from it according to a certain rule specified in advance. If $\left[x_{1}, x_{2} \mid \mathbf{f}\right]$ is selected to be continuous at $x_{1}$ with respect to $x_{2}$, then it is easy to see that $\left[x_{1}, x_{1} \mid \mathbf{f}\right]=\mathbf{f}^{\prime}\left(x_{1}\right)$. Otherwise, the limit

$$
\lim _{t \rightarrow+0}[x, x+t h \mid \mathbf{f}] h=\lim _{t \rightarrow+0} t^{-1}[\mathbf{f}(x+t h)-\mathbf{f}(x)]
$$


(if it exists) may vary depending on $h,\|h\|=1$. In this case, the limit is the directional derivative $\mathbf{f}^{\prime}(x, h)$ of $\mathbf{f}$ in the direction $h$. The following proposition lists for the record obvious properties of the set-valued map $\mathbf{f} \mapsto\left[x_{1}, x_{2} \mid \mathbf{f}\right]$.

Proposition 2.2. (1) $\left[x_{1}, x_{2} \mid \mathbf{f}\right]=\mathbf{f}$ if and only if $\mathbf{f}$ is linear.

(2) $\left[x_{1}, x_{2} \mid \alpha \mathbf{f}_{1}+\beta \mathbf{f}_{2}\right]=\alpha\left[x_{1}, x_{2} \mid \mathbf{f}_{1}\right]+\beta\left[x_{1}, x_{2} \mid \mathbf{f}_{2}\right]$.

(3) If $\mathbf{f}$ is a composition of the operators $\mathbf{f}_{1}, \mathbf{f}_{2}: \mathbf{f}=\mathbf{f}_{1} \circ \mathbf{f}_{2}$, then

$$
\left[x_{1}, x_{2} \mid \mathbf{f}\right]=\left[\mathbf{f}_{2}\left(x_{1}\right), \mathbf{f}_{2}\left(x_{2}\right) \mid \mathbf{f}_{1}\right]\left[x_{1}, x_{2} \mid \mathbf{f}_{2}\right] .
$$

Let $\mathcal{N}$ be the class of nondecreasing concave (and so continuous) functions $\omega:[0, \infty) \rightarrow[0, \infty)$ with $\omega(0)=0$. The typical representatives of this class are the functions $t \mapsto c t^{p}, 0<p \leq 1$.

Definition 2.3. The dd $\left[x_{1}, x_{2} \mid \mathbf{f}\right]$ is said to be $\omega$-regularly continuous on $D$, if an $\omega \in \mathcal{N}$ (call it regularity modulus) is known such that

$$
\begin{aligned}
& \omega^{-1}\left(\min \left\{\left\|\left[x_{1}, x_{2} \mid \mathbf{f}\right]\right\|,\left\|\left[u_{1}, u_{2} \mid \mathbf{f}\right]\right\|\right\}+\left\|\left[x_{1}, x_{2} \mid \mathbf{f}\right]-\left[u_{1}, u_{2} \mid \mathbf{f}\right]\right\|\right) \\
& -\omega^{-1}\left(\min \left\{\left\|\left[x_{1}, x_{2} \mid \mathbf{f}\right]\right\|,\left\|\left[u_{1}, u_{2} \mid \mathbf{f}\right]\right\|\right\}\right) \leq\left\|x_{1}-u_{1}\right\|+\left\|x_{2}-u_{2}\right\|
\end{aligned}
$$

for all $x_{1}, x_{2}, u_{1}, u_{2} \in D$. We say also that it is regularly continuous on $D$, if it has there a regularity modulus.

Concavity is understood in the sense of convex analysis [17], that is $\omega$ is concave if it has convex subgraph $\{(s, t) \mid s \geq 0 \& t \leq \omega(s)\}$. Analogously, $\omega^{-1}$ denotes the function whose closed epigraph $\operatorname{cl}\left\{(s, t) \mid s \geq 0 \& t \geq \omega^{-1}(s)\right\}$ is symmetrical to closure of the subgraph of $\omega$ with respect to the axis $t=s$. Clearly, $\omega^{-1}$ is a convex function on $[0, \infty)$ vanishing at zero, increasing in $[0, \omega(\infty)]$, and equal to $\infty$ for all $s>\omega(\infty)$ (if any).

Being monotone, the function $\omega$ has left- and right-hand derivatives at each $s \geq 0$ (they coincide everywhere except, perhaps, for a countable subset of $[0, \infty))$. In what follows, we denote the right-hand and two-sided derivatives by the usual prime $\left({ }^{\prime}\right)$, whereas the backprime ( ') will symbolize the left-hand derivative.

If $\omega$ is linear: $\omega(t)=c t$, then $(2.1)$ becomes

(2.2) $\left\|\left[x_{1}, x_{2} \mid \mathbf{f}\right]-\left[u_{1}, u_{2} \mid \mathbf{f}\right]\right\| \leq c\left(\left\|x_{1}-u_{1}\right\|+\left\|x_{2}-u_{2}\right\|\right), \forall x_{1}, x_{2}, u_{1}, u_{2} \in D$.

The dd $\left[x_{1}, x_{2} \mid \mathbf{f}\right]$ possessing this property is called Lipschitz continuous. For a discussion on how a regularity modulus can be found for a selected dd the reader is referred to [7].

We conclude this section with an immediate consequence of $\omega$-regular continuity of a dd $\left[x_{1}, x_{2} \mid \mathbf{f}\right]$, which will be of use in the next section.

Lemma $2.4([5])$. If $d d\left[x_{1}, x_{2} \mid \mathbf{f}\right]$ is $\omega$-regularly continuous on $D$, then

$$
\begin{aligned}
& \left|\omega^{-1}\left(\left\|\left[x_{1}, x_{2} \mid \mathbf{f}\right]\right\|\right)-\omega^{-1}\left(\left\|\left[u_{1}, u_{2} \mid \mathbf{f}\right]\right\|\right)\right| \\
\leq & \left\|x_{1}-u_{1}\right\|+\left\|x_{2}-u_{2}\right\|, \quad \forall x_{1}, x_{2}, u_{1}, u_{2} \in D .
\end{aligned}
$$


It follows that

$$
\omega^{-1}\left(\left\|\left[x_{1}, x_{2} \mid \mathbf{f}\right]\right\|\right) \geq\left(\omega^{-1}\left(\left\|\left[u_{1}, u_{2} \mid \mathbf{f}\right]\right\|\right)-\left\|x_{1}-u_{1}\right\|-\left\|x_{2}-u_{2}\right\|\right)^{+},
$$

where the superscript + denotes the nonnegative part of a real number:

$$
r^{+}:=\max \{r, 0\} \text {. }
$$

\section{Majorant generator and convergence lemma}

As seen from (1.5), $\mathbf{A}_{+}\left(\mathbf{f}\left(x_{+}\right)-\mathbf{f}(x)\right)=-\mathbf{A f}(x)=x_{+}-x$ and $\mathbf{A}_{+}$is invertible provided $\mathbf{A}$ is invertible and $x_{+} \neq x$ (that is $\mathbf{f}(x) \neq 0$ ). So, invertibility of $\mathbf{A}_{0}$ implies invertibility of all $\mathbf{A}_{n}$ generated by Broyden's method and the equality

$$
\mathbf{A}_{n}=\left[x_{n}, x_{n-1} \mid \mathbf{f}\right]^{-1} .
$$

If $\mathbf{f}$ is differentiable at $x_{0}$ and the derivative $\mathbf{f}^{\prime}\left(x_{0}\right)$ is boundedly invertible, then the natural choice for $\mathbf{A}_{0}$ is $\mathbf{f}^{\prime}\left(x_{0}\right)^{-1}$. However, we cannot recommend this choice in general, since we do not assume here differentiability of $\mathbf{f}$. What we do assume is that $\mathbf{A}_{0}$ is invertible, so that $\mathbf{A}$ and $\mathbf{f}$ in (1.5) can be replaced by their normalizations $\mathbf{A} \mathbf{A}_{0}^{-1}$ and $\mathbf{A}_{0} \mathbf{f}$ without affecting the method. To save indexation, let us assume (with no loss in generality) that $\mathbf{A}$ and $\mathbf{f}$ are already normalized:

$$
\mathbf{A}_{0}=\left[x_{0}, x_{-1} \mid \mathbf{f}\right]^{-1}=\mathbf{I} .
$$

The current iteration $(x, \mathbf{A})$ induces the triple of reals

$$
\bar{t}:=\left\|x-x_{0}\right\|, \bar{\gamma}:=\left\|x-x_{-}\right\|, \bar{\delta}:=\left\|x_{+}-x\right\| .
$$

Clearly, $\bar{t}_{+}:=\left\|x_{+}-x_{0}\right\| \leq \bar{t}+\bar{\delta}$. Also, $\bar{\gamma}_{+}=\bar{\delta}$. The next lemma relates $\bar{\delta}_{+}:=\left\|x_{++}-x_{+}\right\|=\left\|\mathbf{A}_{+} \mathbf{f}\left(x_{+}\right)\right\|$with the triple $(\bar{t}, \bar{\gamma}, \bar{\delta})$. To make its statement shorter, we use the abbreviations

$$
\alpha:=\omega^{-1}(1-\underline{h}), \bar{\gamma}_{0}:=\left\|x_{0}-x_{-1}\right\|, a:=\alpha-\bar{\gamma}_{0} .
$$

Lemma 3.1. Let the selected $d d\left[x_{1}, x_{2} \mid \mathbf{f}\right]$ of $\mathbf{f}$ be $\omega$-regularly continuous on $D$.

$$
\text { If } \bar{t}_{+}+\bar{t}<a \text {, then }
$$

$$
\bar{\delta}_{+} \leq \bar{\delta}\left(\frac{\omega\left(a-\bar{t}_{+}-\bar{t}+\bar{\delta}+\bar{\gamma}\right)}{\omega\left(a-\bar{t}_{+}-\bar{t}\right)}-1\right) .
$$

Proof. $\bar{\delta}_{+} \leq\left\|\mathbf{A}_{+}\right\| \cdot\left\|\mathbf{f}\left(x_{+}\right)\right\|$. By the Banach lemma on perturbations and in view of $(3.2)$,

(3.4) $\left\|\mathbf{A}_{+}\right\|^{-1} \geq\left\|\mathbf{A}_{0}\right\|^{-1}-\left\|\mathbf{A}_{+}^{-1}-\mathbf{A}_{0}^{-1}\right\| \geq 1-\underline{h}-\left\|\left[x_{+}, x \mid \mathbf{f}\right]-\left[x_{0}, x_{-1} \mid \mathbf{f}\right]\right\|$, where by $(2.1)$

$$
\begin{aligned}
& \left\|\left[x_{+}, x \mid \mathbf{f}\right]-\left[x_{0}, x_{-1} \mid \mathbf{f}\right]\right\| \\
\leq & \omega\left(\min \left\{\omega^{-1}\left(\left\|\left[x_{+}, x \mid \mathbf{f}\right]\right\|-\underline{h}\right), \omega^{-1}\left(\left\|\left[x_{0}, x_{-1} \mid \mathbf{f}\right]\right\|-\underline{h}\right)\right\}+\left\|x_{+}-x_{0}\right\|\right.
\end{aligned}
$$




$$
\left.+\left\|x-x_{-1}\right\|\right)-\omega\left(\min \left\{\omega^{-1}\left(\left\|\left[x_{+}, x \mid \mathbf{f}\right]\right\|-\underline{h}\right), \omega^{-1}\left(\left\|\left[x_{0}, x_{-1} \mid \mathbf{f}\right]\right\|-\underline{h}\right)\right\}\right) .
$$

Because of (2.3) and (3.2),

$$
\begin{aligned}
\omega^{-1}\left(\left\|\left[x_{+}, x \mid \mathbf{f}\right]\right\|-\underline{h}\right) & \geq\left(\omega^{-1}(1-\underline{h})-\left\|x_{+}-x_{0}\right\|-\left\|x-x_{-1}\right\|\right)^{+} \\
& \geq\left(\alpha-\bar{t}_{+}-\bar{t}-\bar{\gamma}_{0}\right)^{+} .
\end{aligned}
$$

So, due to concavity and monotonicity of $\omega$,

$$
\begin{aligned}
& \left\|\left[x_{+}, x \mid \mathbf{f}\right]-\left[x_{0}, x_{-1} \mid \mathbf{f}\right]\right\| \\
\leq & \omega\left(\min \left\{\left(\alpha-\bar{t}_{+}-\bar{t}-\bar{\gamma}_{0}\right)^{+}, \alpha\right\}+\bar{t}_{+}+\bar{t}+\bar{\gamma}_{0}\right) \\
& -\omega\left(\min \left\{\left(\alpha-\bar{t}_{+}-\bar{t}-\bar{\gamma}_{0}\right)^{+}, \alpha\right\}\right) \\
= & \omega\left(\left(\alpha-\bar{t}_{+}-\bar{t}-\bar{\gamma}_{0}\right)^{+}+\bar{t}_{+}+\bar{t}+\bar{\gamma}_{0}\right)-\omega\left(\left(\alpha-\bar{t}_{+}-\bar{t}-\bar{\gamma}_{0}\right)^{+}\right) .
\end{aligned}
$$

If this difference $<1-\underline{h}$, then (3.4) yields

$$
\left\|\mathbf{A}_{+}\right\| \leq \frac{1}{1-\underline{h}-\omega\left(\left(\alpha-\bar{t}_{+}-\bar{t}-\bar{\gamma}_{0}\right)^{+}+\bar{t}_{+}+\bar{t}+\bar{\gamma}_{0}\right)+\omega\left(\left(\alpha-\bar{t}_{+}-\bar{t}-\bar{\gamma}_{0}\right)^{+}\right)} .
$$

The difference $(3.6)<1-\underline{h}=\omega(\alpha)$ if and only if $\bar{t}_{+}+\bar{t}<a$. Hence, this assumption implies

$$
\left\|\mathbf{A}_{+}\right\| \leq \frac{1}{1-\underline{h}-\omega(\alpha)+\omega\left(a-\bar{t}_{+}-\bar{t}\right)}=\frac{1}{\omega\left(a-\bar{t}_{+}-\bar{t}\right)} .
$$

As to $\left\|\mathbf{f}\left(x_{+}\right)\right\|$, note that the application of the classical Sherman-Morrison formula to (1.5) gives

$$
\mathbf{A}_{+}^{-1}=\mathbf{A}^{-1}+\frac{\mathbf{f}\left(x_{+}\right)}{\left\|x_{+}-x\right\|^{2}}\left\langle x_{+}-x, \cdot\right\rangle,
$$

so that $\left\|\mathbf{A}_{+}^{-1}-\mathbf{A}^{-1}\right\|=\left\|\mathbf{f}\left(x_{+}\right)\right\| /\left\|x_{+}-x\right\|$ and $\left\|\mathbf{f}\left(x_{+}\right)\right\|=\bar{\delta}\left\|\left[x_{+}, x \mid \mathbf{f}\right]-\left[x, x_{-} \mid \mathbf{f}\right]\right\|$ (because of (3.1)). As follows from (2.1), the last norm

$$
\begin{aligned}
\leq & \omega\left(\min \left\{\omega^{-1}\left(\left\|\left[x_{+}, x \mid \mathbf{f}\right]\right\|-\underline{h}\right), \omega^{-1}\left(\left\|\left[x, x_{-} \mid \mathbf{f}\right]\right\|-\underline{h}\right)\right\}+\left\|x_{+}-x\right\|\right. \\
& \left.+\left\|x-x_{-}\right\|\right)-\omega\left(\min \left\{\omega^{-1}\left(\left\|\left[x_{+}, x \mid \mathbf{f}\right]\right\|-\underline{h}\right), \omega^{-1}\left(\left\|\left[x, x_{-} \mid \mathbf{f}\right]\right\|-\underline{h}\right)\right\}\right) .
\end{aligned}
$$

As in (3.5),

and so

$$
\begin{aligned}
& \omega^{-1}\left(\left\|\left[x_{+}, x \mid \mathbf{f}\right]\right\|-\underline{h}\right) \geq\left(\alpha-\bar{t}_{+}-\bar{t}-\bar{\gamma}_{0}\right)^{+}=a-\bar{t}_{+}-\bar{t}, \\
& \omega^{-1}\left(\left\|\left[x, x_{-} \mid \mathbf{f}\right]\right\|-\underline{h}\right) \geq\left(\alpha-\bar{t}-\bar{t}_{-}-\bar{\gamma}_{0}\right)^{+}=a-\bar{t}-\bar{t}_{-},
\end{aligned}
$$

$$
\begin{aligned}
\left\|\left[x_{+}, x \mid \mathbf{f}\right]-\left[x, x_{-} \mid \mathbf{f}\right]\right\| \leq & \omega\left(\min \left\{a-\bar{t}_{+}-\bar{t}, a-\bar{t}-\bar{t}_{-}\right\}+\bar{\delta}+\bar{\gamma}\right) \\
& -\omega\left(\min \left\{a-\bar{t}_{+}-\bar{t}, a-\bar{t}-\bar{t}_{-}\right\}\right) \\
= & \omega\left(a-\bar{t}_{+}-\bar{t}+\bar{\delta}+\bar{\gamma}\right)-\omega\left(a-\bar{t}_{+}-\bar{t}\right) .
\end{aligned}
$$


Hence,

$$
\left\|\mathbf{f}\left(x_{+}\right)\right\| \leq \bar{\delta}\left(\omega\left(a-\bar{t}_{+}-\bar{t}+\bar{\delta}+\bar{\gamma}\right)-\omega\left(a-\bar{t}_{+}-\bar{t}\right)\right) .
$$

Together with (3.7) this results in

$$
\bar{\delta}_{+} \leq \bar{\delta}\left(\frac{\omega\left(a-\bar{t}_{+}-\bar{t}+\bar{\delta}+\bar{\gamma}\right)}{\omega\left(a-\bar{t}_{+}-\bar{t}\right)}-1\right)
$$

as claimed.

The lemma suggests the following majorant generator $\mathbf{g}(t, \gamma, \delta)=\left(t_{+}, \gamma_{+}, \delta_{+}\right)$:

$$
\begin{gathered}
t_{+}:=t+\delta, \gamma_{+}:=\delta \\
\delta_{+}:=\delta\left(\frac{\omega\left(a-t_{+}-t+\delta+\gamma\right)}{\omega\left(a-t_{+}-t\right)}-1\right)=\delta\left(\frac{\omega(a-2 t+\gamma)}{\omega(a-2 t-\delta)}-1\right) .
\end{gathered}
$$

We say that the triple $q^{\prime}=\left(t^{\prime}, \gamma^{\prime}, \delta^{\prime}\right)$ majorizes $q=(t, \gamma, \delta)$ (briefly $\left.q \prec q^{\prime}\right)$, if

$$
t \leq t^{\prime} \quad \& \quad \gamma \leq \gamma^{\prime} \& \delta \leq \delta^{\prime}
$$

The lemma states that $\bar{q}_{+} \prec \mathbf{g}(\bar{q})$.

Being fed with the initial triple $q_{0}$, the generator iterates producing a majorant sequence as long as the denominator in (3.10) remains defined:

$$
\&_{n} 2 t_{n}+\delta_{n}<a
$$

Under this condition, we can ensure convergence of the sequence $\left(x_{n}, \mathbf{A}_{n}\right)$ generated by the method (1.5) from the starter $\left(x_{0}, \mathbf{A}_{0}\right)$ to a solution of the system

$$
\mathbf{f}(x)=0 \quad \& \quad \mathbf{A}[x, x \mid \mathbf{f}]=\mathbf{I} .
$$

Lemma 3.2. If $q_{0}$ is such that $\bar{q}_{0} \prec q_{0} \quad \& \quad \underset{n}{\&} 2 t_{n}+\delta_{n}<a$, then

(1) $\& \bar{q}_{n} \prec q_{n}$;

(2) $\gamma_{\infty}=\delta_{\infty}=0 \quad \& \quad \& t_{n} \leq 0.5\left(a-\delta_{n}\right)$;

(3) the sequence $\left(x_{n}, \mathbf{A}_{n}\right)$ remains in the ball $B\left(\left(x_{0}, \mathbf{A}_{0}\right),\left(t_{\infty}, r_{\mathbf{A}}\right)\right)$, where

$$
r_{\mathrm{A}}:=\frac{\omega\left(a-\delta_{0}\right)-\omega\left(a-2 t_{\infty}\right)}{\omega\left(a-\delta_{0}\right) \omega\left(a-2 t_{\infty}\right)}+\frac{\omega\left(a+\gamma_{0}\right)-\omega\left(a-\delta_{0}\right)}{1-\omega\left(a+\gamma_{0}\right)+\omega\left(a-\delta_{0}\right)},
$$

and converges to a solution $\left(x_{\infty}, \mathbf{A}_{\infty}\right)$ of the system (3.11);

(4) $x_{\infty}$ is the only solution of the equation $\mathbf{f}(x)=0$ in the ball $B\left(x_{0}, a-t_{\infty}\right)$;

(5) for all $n=0,1, \ldots$,

$$
\begin{aligned}
& \left\|\mathbf{f}\left(x_{n+1}\right)\right\| \leq \delta_{n}\left(\omega\left(a-2 t_{n}+\gamma_{n}\right)-\omega\left(a-2 t_{n}-\delta_{n}\right)\right), \\
& \Delta_{n}:=\left\|x_{\infty}-x_{n}\right\| \leq t_{\infty}-t_{n}, \\
& \frac{\Delta_{n+1}}{\Delta_{n}} \leq \frac{\omega\left(\Delta_{n-1}\right)}{\omega\left(a-2 t_{n}+\gamma_{n}\right)}
\end{aligned}
$$

(6) all these inequalities are exact in the sense that they hold as equalities for a scalar quadratic polynomial. 
Proof. (1) The generator (3.10) is monotone in the sense that $q \prec q^{\prime} \Longrightarrow \mathbf{g}(q) \prec$ $\mathbf{g}\left(q^{\prime}\right)$. Besides, we have noted above that $\bar{q}_{+} \prec \mathbf{g}(\bar{q})$. Hence, $\bar{q}_{+} \prec \mathbf{g}(q)=q_{+}$. By induction, $\bar{q}_{0} \prec q_{0} \Longrightarrow \&_{n} \bar{q}_{n} \prec q_{n}$.

(2) $2 t_{n}+\delta_{n}<a \Longleftrightarrow t_{n}<0.5\left(a-\delta_{n}\right)$. Thus, the sequence $t_{n}$ is increasing and bounded, so that $t_{\infty}$ is finite. Taking the limits in $t_{n+1}=t_{n}+\delta_{n} \& \gamma_{n+1}=\delta_{n}$ yields $\gamma_{\infty}=\delta_{\infty}=0$.

(3) By (1), \& $\bar{\delta}_{n} \leq \delta_{n}$ and so

$\left\|x_{n+m}-x_{n}\right\| \leq \sum_{k=n}^{n+m-1}\left\|x_{k+1}-x_{k}\right\|=\sum_{k=n}^{n+m-1} \bar{\delta}_{k} \leq \sum_{k=n}^{n+m-1} \delta_{k}<\sum_{k=n}^{\infty} \delta_{k}=t_{\infty}-t_{n}$.

This shows that $x_{n}$ is a Cauchy sequence and so converges: $\exists \lim x_{n}=: x_{\infty}$. Setting $n=0$ results in $x_{n} \in B\left(x_{0}, t_{\infty}\right)$, while forcing $m$ to $\infty$ yields $\left\|x_{\infty}-x_{n}\right\| \leq$ $t_{\infty}-t_{n}$. Moreover, in view of (3.9) and because of concavity of $\omega$,

$$
\begin{aligned}
\left\|\mathbf{f}\left(x_{n+1}\right)\right\| & \leq \bar{\delta}_{n}\left(\omega\left(a-\bar{t}_{n+1}-\bar{t}_{n}+\bar{\delta}_{n}+\bar{\gamma}_{n}\right)-\omega\left(a-\bar{t}_{n+1}-\bar{t}_{n}\right)\right) \\
& \leq \delta_{n}\left(\omega\left(a-t_{n+1}-t_{n}+\delta_{n}+\gamma_{n}\right)-\omega\left(a-t_{n+1}-t_{n}\right)\right) \\
& =\delta_{n}\left(\omega\left(a-2 t_{n}+\gamma_{n}\right)-\omega\left(a-2 t_{n}-\delta_{n}\right)\right) \rightarrow 0,
\end{aligned}
$$

so that $\mathbf{f}\left(x_{\infty}\right)=0$. Consider now the operators $\mathbf{A}_{m+n}-\mathbf{A}_{n}$. By the classic Banach lemma (see, for example, [12, Theorem 1.2.1]),

$$
\left\|\mathbf{A}_{m+n}-\mathbf{A}_{n}\right\| \leq \frac{\left\|\mathbf{A}_{n}\right\|^{2}\left\|\mathbf{A}_{m+n}^{-1}-\mathbf{A}_{n}^{-1}\right\|}{1-\left\|\mathbf{A}_{n}\right\| \cdot\left\|\mathbf{A}_{m+n}^{-1}-\mathbf{A}_{n}^{-1}\right\|},
$$

where $\left\|\mathbf{A}_{m+n}^{-1}-\mathbf{A}_{n}^{-1}\right\| \leq \sum_{k=n}^{m+n-1}\left\|\mathbf{A}_{k+1}^{-1}-\mathbf{A}_{k}^{-1}\right\|$. By (3.1) and (3.8),

$$
\begin{aligned}
\left\|\mathbf{A}_{k+1}^{-1}-\mathbf{A}_{k}^{-1}\right\| & =\left\|\left[x_{k+1}, x_{k} \mid \mathbf{f}\right]-\left[x_{k}, x_{k-1} \mid \mathbf{f}\right]\right\| \\
& \leq \omega\left(a-2 t_{k}+\gamma_{k}\right)-\omega\left(a-2 t_{k}-\delta_{k}\right) .
\end{aligned}
$$

According to (3.10), $t_{k}=t_{k-1}+\delta_{k-1}$ and $\gamma_{k}=\delta_{k-1}$. Therefore, $a-2 t_{k}+\gamma_{k}=$ $a-2 t_{k-1}-\delta_{k-1},\left\|\mathbf{A}_{k+1}^{-1}-\mathbf{A}_{k}^{-1}\right\| \leq \omega\left(a-2 t_{k-1}-\delta_{k-1}\right)-\omega\left(a-2 t_{k}-\delta_{k}\right)$ and

$$
\begin{aligned}
\left\|\mathbf{A}_{m+n}^{-1}-\mathbf{A}_{n}^{-1}\right\| & \leq \sum_{k=n}^{m+n-1}\left(\omega\left(a-2 t_{k-1}-\delta_{k-1}\right)-\omega\left(a-2 t_{k}-\delta_{k}\right)\right) \\
& =\omega\left(a-2 t_{n-1}-\delta_{n-1}\right)-\omega\left(a-2 t_{m+n-1}-\delta_{m+n-1}\right) \\
& <\omega\left(a-2 t_{n-1}-\delta_{n-1}\right)-\omega\left(a-2 t_{\infty}\right) .
\end{aligned}
$$

This shows that $\mathbf{A}_{n}^{-1}$ is a Cauchy sequence in the Banach space $\mathcal{L}(\mathbb{X}, \mathbb{H})$ of bounded linear operators from $\mathbb{X}$ to $\mathbb{H}$ and so converges to a limit $\mathbf{A}_{\infty}^{-1}$. As $\mathbf{A}_{n}^{-1}=\left[x_{n}, x_{n-1} \mid \mathbf{f}\right]$, taking limits yields $\mathbf{A}_{\infty}^{-1}=\left[x_{\infty}, x_{\infty} \mid \mathbf{f}\right]$ or $\mathbf{A}_{\infty}\left[x_{\infty}, x_{\infty} \mid \mathbf{f}\right]$ $=\mathbf{I}$. Besides, (3.14) implies $\left\|\mathbf{A}_{m+1}^{-1}-\mathbf{A}_{1}^{-1}\right\|<\omega\left(a-\delta_{0}\right)-\omega\left(a-2 t_{\infty}\right)$ and so

$$
\left\|\mathbf{A}_{m+1}-\mathbf{A}_{1}\right\|<\frac{\left\|\mathbf{A}_{1}\right\|^{2}\left(\omega\left(a-\delta_{0}\right)-\omega\left(a-2 t_{\infty}\right)\right)}{1-\left\|\mathbf{A}_{1}\right\|\left(\omega\left(a-\delta_{0}\right)-\omega\left(a-2 t_{\infty}\right)\right)},
$$


where $\left\|\mathbf{A}_{1}\right\| \leq 1 / \omega\left(a-\delta_{0}\right)$ by (3.7). It follows that

$$
\left\|\mathbf{A}_{m+1}-\mathbf{A}_{1}\right\|<\frac{\omega\left(a-\delta_{0}\right)-\omega\left(a-2 t_{\infty}\right)}{\omega\left(a-\delta_{0}\right) \omega\left(a-2 t_{\infty}\right)} .
$$

Also, as seen from (3.13) and (3.2),

$$
\left\|\mathbf{A}_{1}-\mathbf{A}_{0}\right\| \leq \frac{\left\|\mathbf{A}_{1}^{-1}-\mathbf{A}_{0}^{-1}\right\|}{1-\left\|\mathbf{A}_{1}^{-1}-\mathbf{A}_{0}^{-1}\right\|},
$$

where $\left\|\mathbf{A}_{1}^{-1}-\mathbf{A}_{0}^{-1}\right\| \leq \omega\left(a+\gamma_{0}\right)-\omega\left(a-\delta_{0}\right)$ by (3.8). Therefore,

$$
\left\|\mathbf{A}_{1}-\mathbf{A}_{0}\right\| \leq \frac{\omega\left(a+\gamma_{0}\right)-\omega\left(a-\delta_{0}\right)}{1-\omega\left(a+\gamma_{0}\right)+\omega\left(a-\delta_{0}\right)}
$$

and

$$
\begin{aligned}
\left\|\mathbf{A}_{m+1}-\mathbf{A}_{0}\right\| & \leq\left\|\mathbf{A}_{m+1}-\mathbf{A}_{1}\right\|+\left\|\mathbf{A}_{1}-\mathbf{A}_{0}\right\| \\
& \leq \frac{\omega\left(a-\delta_{0}\right)-\omega\left(a-2 t_{\infty}\right)}{\omega\left(a-\delta_{0}\right) \omega\left(a-2 t_{\infty}\right)}+\frac{\omega\left(a+\gamma_{0}\right)-\omega\left(a-\delta_{0}\right)}{1-\omega\left(a+\gamma_{0}\right)+\omega\left(a-\delta_{0}\right)}=: r_{\mathbf{A}} .
\end{aligned}
$$

(4) Let $x_{*}$ be another solution of the equation $\mathbf{f}(x)=0$. Then $0=\mathbf{f}\left(x_{*}\right)-$ $\mathbf{f}\left(x_{\infty}\right)=\left[x_{*}, x_{\infty} \mid \mathbf{f}\right]\left(x_{*}-x_{\infty}\right)$, which shows that the dd $\left[x_{*}, x_{\infty} \mid \mathbf{f}\right]$ is not invertible and so $1 \leq\left\|\mathbf{I}-\left[x_{*}, x_{\infty} \mid \mathbf{f}\right]\right\|=\left\|\left[x_{0}, x_{-1} \mid \mathbf{f}\right]-\left[x^{*}, x_{\infty} \mid \mathbf{f}\right]\right\|$. By (2.1), this norm

$$
\begin{aligned}
& \leq \omega\left(\min \left\{\omega^{-1}(1-\underline{h}),\left(\omega^{-1}\left(\left\|\left[x_{*}, x_{\infty} \mid \mathbf{f}\right]\right\|-\underline{h}\right)\right\}+\left\|x^{*}-x_{0}\right\|+\bar{t}_{\infty}+\bar{\gamma}_{0}\right)-\right. \\
& \omega\left(\min \left\{\omega^{-1}(1-\underline{h}),\left(\omega^{-1}\left(\left\|\left[x_{*}, x_{\infty} \mid \mathbf{f}\right]\right\|-\underline{h}\right)\right\}\right) .\right.
\end{aligned}
$$

Here,

$$
\begin{aligned}
\omega^{-1}\left(\left\|\left[x_{*}, x_{\infty} \mid \mathbf{f}\right]\right\|-\underline{h}\right) & \geq\left(\omega^{-1}(1-\underline{h})-\left\|x_{*}-x_{0}\right\|-\bar{t}_{\infty}-\bar{\gamma}_{0}\right)^{+} \\
& \geq\left(\alpha-\left\|x_{*}-x_{0}\right\|-t_{\infty}-\bar{\gamma}_{0}\right)^{+}
\end{aligned}
$$

by (2.3). Therefore,

$$
\begin{aligned}
1 \leq & \omega\left(\min \left\{\alpha,\left(\alpha-\left\|x_{*}-x_{0}\right\|-t_{\infty}-\bar{\gamma}_{0}\right)^{+}\right\}+\left\|x_{*}-x_{0}\right\|+t_{\infty}+\bar{\gamma}_{0}\right) \\
& -\omega\left(\min \left\{\alpha,\left(\alpha-\left\|x_{*}-x_{0}\right\|-t_{\infty}-\bar{\gamma}_{0}\right)^{+}\right\}\right) \\
= & \omega\left(\left(a-\left\|x_{*}-x_{0}\right\|-t_{\infty}\right)^{+}+\left\|x_{*}-x_{0}\right\|+t_{\infty}+\bar{\gamma}_{0}\right)-\omega\left(\left(a-\left\|x_{*}-x_{0}\right\|-t_{\infty}\right)^{+}\right) \\
\leq & \omega\left(\left\|x_{*}-x_{0}\right\|+t_{\infty}+\bar{\gamma}_{0}\right)
\end{aligned}
$$

(because of concavity of $\omega$ ) and $\left\|x_{*}-x_{0}\right\| \geq \omega^{-1}(1)-\bar{\gamma}_{0}-t_{\infty} \geq a-t_{\infty} \geq 0.5 a$.

(5) The bounds for $\mathbf{f}\left(x_{n+1}\right)$ and $\Delta_{n}$ have been proved above. It remains to obtain one for the ratio $\Delta_{n+1} / \Delta_{n}$. As

$$
\begin{aligned}
x_{n+1}-x_{\infty} & =x_{n}-x_{\infty}-\mathbf{A}_{n} \mathbf{f}\left(x_{n}\right) \\
& =\mathbf{A}_{n}\left[x_{n}, x_{n-1} \mid \mathbf{f}\right]\left(x_{n}-x_{\infty}\right)-\mathbf{A}_{n}\left(\mathbf{f}\left(x_{n}\right)-\mathbf{f}\left(x_{\infty}\right)\right) \\
& =\mathbf{A}_{n}\left[x_{n}, x_{n-1} \mid \mathbf{f}\right]\left(x_{n}-x_{\infty}\right)-\mathbf{A}_{n}\left[x_{n}, x_{\infty} \mid \mathbf{f}\right]\left(x_{n}-x_{\infty}\right) \\
& =\mathbf{A}_{n}\left(\left[x_{n}, x_{n-1} \mid \mathbf{f}\right]-\left[x_{n}, x_{\infty} \mid \mathbf{f}\right]\right)\left(x_{n}-x_{\infty}\right),
\end{aligned}
$$


we have the inequality $\Delta_{n+1} \leq \Delta_{n}\left\|\mathbf{A}_{n}\right\|\left\|\left[x_{n}, x_{n-1} \mid \mathbf{f}\right]-\left[x_{n}, x_{\infty} \mid \mathbf{f}\right]\right\|$. By (3.7),

$$
\left\|\mathbf{A}_{n}\right\| \leq \frac{1}{\omega\left(a-\bar{t}_{n}-\bar{t}_{n-1}\right)} \leq \frac{1}{\omega\left(a-t_{n}-t_{n-1}\right)}=\frac{1}{\omega\left(a-2 t_{n}+\gamma_{n}\right)} .
$$

Besides, by (2.1)

$$
\begin{aligned}
& \left\|\left[x_{n}, x_{n-1} \mid \mathbf{f}\right]-\left[x_{n}, x_{\infty} \mid \mathbf{f}\right]\right\| \\
\leq & \omega\left(\min \left\{\omega^{-1}\left(\|\left[x_{n}, x_{n-1} \mid \mathbf{f}\right]-\underline{h}\right), \omega^{-1}\left(\|\left[x_{n}, x_{\infty} \mid \mathbf{f}\right]-\underline{h}\right)\right\}+\left\|x_{n-1}-x_{\infty}\right\|\right) \\
& -\omega\left(\min \left\{\omega^{-1}\left(\|\left[x_{n}, x_{n-1} \mid \mathbf{f}\right]-\underline{h}\right), \omega^{-1}\left(\|\left[x_{n}, x_{\infty} \mid \mathbf{f}\right]-\underline{h}\right)\right\}\right),
\end{aligned}
$$

where $\omega^{-1}\left(\|\left[x_{n}, x_{\infty} \mid \mathbf{f}\right]-\underline{h}\right) \geq\left(\omega^{-1}\left(\|\left[x_{n}, x_{n-1} \mid \mathbf{f}\right]-\underline{h}\right)-\left\|x_{n-1}-x_{\infty}\right\|\right)^{+}$by (2.3). So,

$$
\begin{aligned}
& \left\|\left[x_{n}, x_{n-1} \mid \mathbf{f}\right]-\left[x_{n}, x_{\infty} \mid \mathbf{f}\right]\right\| \\
\leq & \omega\left(\left(\omega^{-1}\left(\|\left[x_{n}, x_{n-1} \mid \mathbf{f}\right]-\underline{h}\right)-\Delta_{n-1}\right)^{+}+\Delta_{n-1}\right) \\
& -\omega\left(\left(\omega^{-1}\left(\|\left[x_{n}, x_{n-1} \mid \mathbf{f}\right]-\underline{h}\right)-\Delta_{n-1}\right)^{+}\right) \\
\leq & \omega\left(\Delta_{n-1}\right) .
\end{aligned}
$$

Thus, $\Delta_{n+1} \leq \Delta_{n} \frac{\omega\left(\Delta_{n-1}\right)}{\omega\left(a-2 t_{n}+\gamma_{n}\right)}$.

(6) For any scalar function $f$ with $\omega$-regularly continuous dd's, the application of the method (1.5) produces the sequence of triples

$$
\left(\bar{t}_{n}, \bar{\gamma}_{n}, \bar{\delta}_{n}\right)=\left(x_{n}-x_{0}, x_{n}-x_{n-1}, x_{n+1}-x_{n}\right) .
$$

If $(t, \gamma, \delta)=\left(x-x_{0}, x-x_{-}, x_{+}-x\right)$, then $t_{+}:=t+\delta=x-x_{0}+x_{+}-x=x_{+}-x_{0}$, $\gamma_{+}:=\delta=x_{+}-x$, and

$$
\delta_{+}:=\delta\left(\frac{\omega(a-2 t+\gamma)}{\omega(a-2 t-\delta)}-1\right)=\left(x_{+}-x\right)\left(\frac{\omega\left(a+2 x_{0}-x-x_{-}\right)}{\omega\left(a+2 x_{0}-x_{+}-x\right)}-1\right) .
$$

So, $(t, \gamma, \delta)=\left(x-x_{0}, x-x_{-}, x_{+}-x\right)$ implies $\left(t_{+}, \gamma_{+}, \delta_{+}\right)=\left(x_{+}-x_{0}, x_{+}-\right.$ $\left.x, x_{++}-x_{+}\right)$if and only if

$$
\left(x_{+}-x\right)\left(\frac{\omega\left(a+2 x_{0}-x-x_{-}\right)}{\omega\left(a+2 x_{0}-x_{+}-x\right)}-1\right)=-\frac{f\left(x_{+}\right)}{\left[x_{+}, x \mid f\right]} .
$$

For linear $\omega$ (the case of Lipschitz continuity of the dd), this equation becomes

$$
\frac{\left(x_{+}-x\right)\left(x_{+}-x_{-}\right)}{b-x-x_{+}}=-\frac{f\left(x_{+}\right)}{\left[x_{+}, x \mid f\right]}, \quad b:=a+2 x_{0} .
$$

This functional equation is solved by the quadratic polynomial $f(x):=x^{2}-$ $b x+c$. Indeed, for it $\left[x_{+}, x \mid f\right]=x_{+}+x-b$,

$$
x_{+}-x=-\frac{f(x)}{\left[x, x_{-} \mid f\right]}=-\frac{f(x)}{x+x_{-}-b}=\frac{f(x)}{b-2 x+\gamma},
$$


and $x_{+}-x_{-}=\gamma+\frac{f(x)}{b-2 x+\gamma}$, so that (3.15) can be rewritten as

$$
\begin{aligned}
& \frac{f(x)}{b-2 x+\gamma}\left(\gamma+\frac{f(x)}{b-2 x+\gamma}\right) \\
= & f\left(x+\frac{f(x)}{b-2 x+\gamma}\right) \\
= & \left(x+\frac{f(x)}{b-2 x+\gamma}\right)^{2}-b\left(x+\frac{f(x)}{b-2 x+\gamma}\right)+c \\
= & \frac{(f(x)+x(b-2 x+\gamma))^{2}-b(f(x)+x(b-2 x+\gamma))(b-2 x+\gamma)+c(b-2 x+\gamma)^{2}}{(b-2 x+\gamma)^{2}}
\end{aligned}
$$

or, equivalently,

$$
\begin{aligned}
& f(x)[f(x)+\gamma(b-2 x+\gamma)] \\
= & {[f(x)+x(b-2 x+\gamma)]^{2}-b[f(x)+x(b-2 x+\gamma)](b-2 x+\gamma) } \\
& +c(b-2 x+\gamma)^{2} .
\end{aligned}
$$

Expanding the expression on the right yields

$$
\begin{aligned}
& f(x)+2 x f(x)(b-2 x+\gamma)+x^{2}(b-2 x+\gamma)^{2}-b f(x)(b-2 x+\gamma) \\
& -b x(b-2 x+\gamma)^{2}+c(b-2 x+\gamma)^{2} \\
= & \left(x^{2}-b x+c\right)(b-2 x+\gamma)^{2}-f(x)(b-2 x)(b-2 x+\gamma)+f(x)^{2} \\
= & f(x)\left[(b-2 x+\gamma)^{2}-(b-2 x)(b-2 x+\gamma)+f(x)\right] \\
= & f(x)[\gamma(b-2 x+\gamma)+f(x)],
\end{aligned}
$$

the expression on the left. Therefore, $(t, \gamma, \delta)=(\bar{t}, \bar{\gamma}, \bar{\delta})$ implies $\left(t_{+}, \gamma_{+}, \delta_{+}\right)=$ $\left(\bar{t}_{+}, \bar{\gamma}_{+}, \bar{\delta}_{+}\right)$and by induction

$$
\begin{aligned}
& \gamma_{0}=x_{0}-x_{-1} \& \delta_{0}=-\frac{f\left(x_{0}\right)}{\left[x_{0}, x_{-1} \mid f\right]} \\
\Longrightarrow & \underset{n}{\&}\left(t_{n}, \gamma_{n}, \delta_{n}\right)=\left(x_{n}-x_{0}, x_{n}-x_{n-1}, x_{n+1}-x_{n}\right) .
\end{aligned}
$$

Hence the claim.

\section{Convergence theorem}

As Lemma 3.2 shows, in order to guarantee convergence of $x_{n}$, one has to choose such starters $x_{0}, x_{-1}, \mathbf{A}_{0}, q_{0}$ that $\bar{q}_{0} \prec q_{0} \quad \& \quad \underset{n}{\&} 2 t_{n}+\delta_{n}<a$. So, our next task is to get a precise description of the set of all $q_{0}$ that result in $\underset{n}{\&} 2 t_{n}+\delta_{n}<a$ (the convergence domain of the generator (3.10)).

Proposition 4.1. Let $\left(t_{n}, \gamma_{n}, \delta_{n}\right)$ be the sequence generated by the generator (3.10) from the starter $\left(t_{0}, \gamma_{0}, \delta_{0}\right)$. Then 
(1) $\underset{n}{\&} 2 t_{n}+\delta_{n}<a \Longleftrightarrow \delta_{0} \leq f_{\infty}\left(t_{0}, \gamma_{0}\right)$, where

$$
f_{0}(t, \gamma):=\omega^{-1}(1-\underline{h})-2 t-\gamma
$$

for all $(t, \gamma)$ with $2 t+\gamma \leq \omega^{-1}(1-\underline{h})$ and $f_{n+1}(t, \gamma)$ the unique solution for $\delta$ of the equation

$$
f_{n}(t+\delta, \delta)=\delta\left(\frac{\omega(a-2 t+\gamma)}{\omega(a-2 t-\delta)}-1\right) .
$$

(2) The function $f_{\infty}$ is a solution of the system

$$
x(t+x(t, \gamma), x(t, \gamma))=x(t, \gamma)\left(\frac{\omega(a-2 t+\gamma)}{\omega(a-2 t-x(t, \gamma))}-1\right) \quad \& \quad x\left(t_{\infty}, \gamma\right)=0 \text {. }
$$

(3) The function $I(t, \gamma, \delta):=f_{\infty}(t, \gamma)-\delta$ is an invariant of the generator $(3.10)$.

Proof. (1) $2 t_{n}+\delta_{n}<a \Longleftrightarrow \delta_{n}<a-2 t_{n}=: f_{0}\left(t_{n}, \gamma_{n}\right)$. Suppose that for some $k \geq 0 \delta_{n-k}<f_{k}\left(t_{n-k}, \gamma_{n-k}\right)$, where $f_{k}$ is decreasing with respect to the first argument to $f_{k}\left(t_{\infty}, \gamma\right)=0$ and not increasing with respect to the second. Using (3.10), rewrite this inequality as

$$
\delta_{n-k-1}\left(\frac{\omega\left(a-2 t_{n-k-1}+\gamma_{n-k-1}\right)}{\omega\left(a-2 t_{n-k-1}-\delta_{n-k-1}\right)}-1\right)<f_{k}\left(t_{n-k-1}+\delta_{n-k-1}, \delta_{n-k-1}\right)
$$

or, equivalently, as $F_{k}\left(t_{n-k-1}, \gamma_{n-k-1}, \delta_{n-k-1}\right)>0$, where

$$
F_{k}(t, \gamma, \delta):=f_{k}(t+\delta, \delta)-\delta\left(\frac{\omega(a-2 t+\gamma)}{\omega(a-2 t-\delta)}-1\right)
$$

The function $F_{k}$ is decreasing in $\delta \in\left[0, t_{\infty}-t\right]$ from $F_{k}(t, \gamma, 0)=f_{k}(t, 0)>0$ to

$$
\begin{aligned}
F_{k}\left(t, \gamma, t_{\infty}-t\right) & =f_{k}\left(t+t_{\infty}-t, t_{\infty}-t\right)-\left(t_{\infty}-t\right)\left(\frac{\omega(a-2 t+\gamma)}{\omega\left(a-2 t-t_{\infty}+t\right)}-1\right) \\
& =f_{k}\left(t_{\infty}, t_{\infty}-t\right)-\left(t_{\infty}-t\right)\left(\frac{\omega(a-2 t+\gamma)}{\omega\left(a-t-t_{\infty}\right)}-1\right) \\
& =-\left(t_{\infty}-t\right)\left(\frac{\omega(a-2 t+\gamma)}{\omega\left(a-t-t_{\infty}\right)}-1\right)<0 .
\end{aligned}
$$

Therefore, the equation $F_{k}(t, \gamma, \delta)=0$ is uniquely solvable for $\delta \in\left(0, t_{\infty}-t\right)$. We denote the solution $f_{k+1}(t, \gamma)$ :

$$
F_{k}\left(t, \gamma, f_{k+1}(t, \gamma)\right)=0 .
$$

The function $f_{k+1}$ is decreasing in $t \in\left[0, t_{\infty}\right)$. To see it, note that it follows from the induction hypothesis and properties of $\omega$, that $F_{k}$ is decreasing in both $t$ and $\delta$. So,

$$
\begin{aligned}
t<t^{\prime} & \Longrightarrow F_{k}\left(t, \gamma, f_{k+1}(t, \gamma)\right)=0=F_{k}\left(t^{\prime}, \gamma, f_{k+1}\left(t^{\prime}, \gamma\right)\right)<F_{k}\left(t, \gamma, f_{k+1}\left(t^{\prime}, \gamma\right)\right) \\
& \Longrightarrow f_{k+1}\left(t^{\prime}, \gamma\right)<f_{k+1}(t, \gamma)
\end{aligned}
$$


Similarly,

$$
\begin{aligned}
\gamma<\gamma^{\prime} & \Longrightarrow F_{k}\left(t, \gamma, f_{k+1}(t, \gamma)\right)=0=F_{k}\left(t, \gamma^{\prime}, f_{k+1}\left(t, \gamma^{\prime}\right)\right)<F_{k}\left(t, \gamma, f_{k+1}\left(t, \gamma^{\prime}\right)\right) \\
& \Longrightarrow f_{k+1}\left(t, \gamma^{\prime}\right)<f_{k+1}(t, \gamma) .
\end{aligned}
$$

Besides, by the induction hypothesis, $F_{k}\left(t_{\infty}, \gamma, 0\right)=f_{k}\left(t_{\infty}, 0\right)=0$, which means according to $(4.3)$ that $f_{k+1}\left(t_{\infty}, \gamma\right)=0$. Thus,

$$
\delta_{n-k}<f_{k}\left(t_{n-k}, \gamma_{n-k}\right) \Longrightarrow \delta_{n-k-1}<f_{k+1}\left(t_{n-k-1}, \gamma_{n-k-1}\right) .
$$

By induction, $2 t_{n}+\delta_{n}<a \Longleftrightarrow \delta_{0}<f_{n}\left(t_{0}, \gamma_{0}\right)$ and

$$
\&_{n} 2 t_{n}+\delta_{n}<a \Longleftrightarrow \delta_{0} \leq \inf _{n} f_{n}\left(t_{0}, \gamma_{0}\right)
$$

The sequence $f_{n}$ is pointwise decreasing:

$$
\underset{n}{\&} f_{n+1}(t, \gamma)<f_{n}(t, \gamma)
$$

This is proved inductively. By definition,

$$
f_{1}(t, \gamma) \in\left(0, t_{\infty}-t\right) \subset(0,0.5 a-t)=\left(0,0.5 f_{0}(t, \gamma)\right)
$$

so that $f_{1}(t, \gamma)<f_{0}(t, \gamma)$. Suppose now that $f_{n}(t, \gamma)<f_{n-1}(t, \gamma)$ for some $n \geq 1$. Then by (4.3) and (4.2)

$$
\begin{aligned}
& F_{n-1}\left(t, \gamma, f_{n}(t, \gamma)\right)=0=F_{n}\left(t, \gamma, f_{n+1}(t, \gamma)\right) \\
= & f_{n}\left(t+f_{n+1}(t, \gamma), f_{n+1}(t, \gamma)\right)-f_{n+1}(t, \gamma)\left(\frac{\omega(a-2 t+\gamma)}{\omega\left(a-2 t-f_{n+1}(t, \gamma)\right)}-1\right) \\
< & f_{n-1}\left(t+f_{n+1}(t, \gamma), f_{n+1}(t, \gamma)\right)-f_{n+1}(t, \gamma)\left(\frac{\omega(a-2 t+\gamma)}{\omega\left(a-2 t-f_{n+1}(t, \gamma)\right)}-1\right) \\
= & F_{n-1}\left(t, \gamma, f_{n+1}(t, \gamma)\right) \Longrightarrow f_{n+1}(t, \gamma)<f_{n}(t, \gamma),
\end{aligned}
$$

because $F_{n-1}$ is decreasing with respect to the third argument. Thus,

$$
f_{n}(t, \gamma)<f_{n-1}(t, \gamma) \Longrightarrow f_{n+1}(t, \gamma)<f_{n}(t, \gamma)
$$

By induction, (4.4) is proved. It follows that $\inf _{n} f_{n}\left(t_{0}, \gamma_{0}\right)=f_{\infty}\left(t_{0}, \gamma_{0}\right)$ and

$$
\underset{n}{\&} 2 t_{n}+\delta_{n}<a \Longleftrightarrow \delta_{0} \leq f_{\infty}\left(t_{0}, \gamma_{0}\right)
$$

(2) Since the sequence $f_{n}$ converges, we can take limits in (4.3) to get $F_{\infty}\left(t, \gamma, f_{\infty}(t, \gamma)\right)=0$, that is

$$
f_{\infty}\left(t+f_{\infty}(t, \gamma), f_{\infty}(t, \gamma)\right)=f_{\infty}(t, \gamma)\left(\frac{\omega(a-2 t+\gamma)}{\omega\left(a-2 t-f_{\infty}(t, \gamma)\right)}-1\right)
$$

Besides, we have seen above that $f_{k}\left(t_{\infty}, \gamma\right)=0 \Longrightarrow f_{k+1}\left(t_{\infty}, \gamma\right)=0$, so that $f_{\infty}\left(t_{\infty}, \gamma\right)=0$. Hence, $f_{\infty}$ satisfies (4.1). 
(3) It suffices to show that $I(t, \gamma, \delta)=0 \Longrightarrow I\left(t_{+}, \gamma_{+}, \delta_{+}\right)=0$. Indeed, $I(t, \gamma, \delta)=0 \Longrightarrow f_{\infty}(t, \gamma)=\delta$, so that

$$
\begin{aligned}
& I\left(t_{+}, \gamma_{+}, \delta_{+}\right)=f_{\infty}\left(t_{+}, \gamma_{+}\right)-\delta_{+}=f_{\infty}(t+\delta, \delta)-\delta\left(\frac{\omega(a-2 t+\gamma)}{\omega(a-2 t-\delta)}-1\right) \\
= & f_{\infty}\left(t+f_{\infty}(t, \gamma), f_{\infty}(t, \gamma)\right)-f_{\infty}(t, \gamma)\left(\frac{\omega(a-2 t+\gamma)}{\omega\left(a-2 t-f_{\infty}(t, \gamma)\right)}-1\right)=0
\end{aligned}
$$

by (2).

For linear $\omega(\omega(t)=c t)$, the generator (3.10) simplifies:

$$
t_{+}:=t+\delta, \gamma_{+}:=\delta, \delta_{+}:=\delta \frac{\gamma+\delta}{a-2 t-\delta},
$$

where $a=c^{-1}-\left\|x_{0}-x_{-1}\right\|$. Consequently, the proposition can be stated more rigorously:

Proposition 4.2. (1) The function $I(t, \gamma, \delta):=(a-2 t)^{2}-4 \delta(a-2 t+\gamma)$ is an invariant of the generator (4.5).

(2) The sequence $\left(t_{n}, \gamma_{n}, \delta_{n}\right)$, generated by the generator (4.5) from the starter $\left(0, \gamma_{0}, \delta_{0}\right)$, converges $\left(t_{\infty}<\infty\right)$ if and only if

$$
\left.I_{0}:=I\left(0, \gamma_{0}, \delta_{0}\right)=\left(c^{-1}-\gamma_{0}\right)^{2}-4 c^{-1} \delta_{0}\right) \geq 0 .
$$

In this case,

$\underset{n}{\&} 2 t_{n}+\delta_{n}<a \Longleftrightarrow t_{n}=0.5\left(c^{-1}-\gamma_{0}\right)-\delta_{n}-\sqrt{\delta_{n}\left(\gamma_{n}+\delta_{n}\right)+0.25 I_{0}}=: f\left(\gamma_{n}, \delta_{n}\right)$.

(3) The function $f$ is a solution of the system

$$
x\left(\delta, \delta \frac{\gamma+\delta}{a-2 x(\gamma, \delta)-\delta}\right)=x(\gamma, \delta)+\delta \quad \& \quad x(0,0)=t_{\infty} .
$$

Proof. (1) By (4.5),

$$
\begin{aligned}
I\left(t_{+}, \gamma_{+}, \delta_{+}\right) & =\left(a-2 t_{+}\right)^{2}-4 \delta_{+}\left(a-2 t_{+}+\gamma_{+}\right) \\
& =(a-2 t-2 \delta)^{2}-4 \delta \frac{\delta+\gamma}{a-2 t-2 \delta+\delta}(a-2 t-2 \delta+\delta) \\
& =(a-2 t)^{2}-4 \delta(a-2 t)+4 \delta^{2}-4 \delta(\delta+\gamma) \\
& =(a-2 t)^{2}-4 \delta(a-2 t)-4 \delta \gamma=I(t, \gamma, \delta) .
\end{aligned}
$$

(2) If $t_{\infty}<\infty$, then $\gamma_{n+1}=\delta_{n}=t_{n+1}-t_{n} \rightarrow 0$. Then by (1), $I\left(0, \gamma_{0}, \delta_{0}\right)=$ $I\left(t_{\infty}, 0,0\right)$, i.e., $a^{2}-4 \delta_{0}\left(a+\gamma_{0}\right)=\left(a-2 t_{\infty}\right)^{2} \geq 0$. Conversely, if $I_{0} \geq 0$, then $\&_{n} I\left(t_{n}, \gamma_{n}, \delta_{n}\right)=I_{0} \geq 0$, whence

$$
t_{n}=0.5\left(a-\delta_{n}-\sqrt{\delta_{n}^{2}+\gamma_{n} \delta_{n}+0.25 I_{0}}\right) \leq 0.5\left(a-\sqrt{I_{0}}\right)
$$

and $t_{\infty}=0.5\left(a-\sqrt{I_{0}}\right)=0.5\left(c^{-1}-\gamma_{0}-\sqrt{I_{0}}\right)<\infty$. 
(3) By $(1), I(t, \gamma, \delta)=\left(a-2 t_{\infty}\right)^{2}=I\left(t_{+}, \gamma_{+}, \delta_{+}\right)$

$\Longleftrightarrow t=0.5 a-\delta-\sqrt{\delta(\gamma+\delta)+\left(0.5 a-t_{\infty}\right)^{2}}=: f(\gamma, \delta) \quad \& \quad t_{+}=f\left(\gamma_{+}, \delta_{+}\right)$

$\Longrightarrow f(\gamma, \delta)+\delta=f\left(\delta, \delta \frac{\gamma+\delta}{a-2 f(\gamma, \delta)-\delta}\right)$.

Besides, $f(\gamma, \delta)=0.5 a-\delta-\sqrt{\delta(\gamma+\delta)+\left(0.5 a-t_{\infty}\right)^{2}} \Longrightarrow f(0,0)=t_{\infty}$.

Now, to get the convergence theorem for operators with $\omega$-regularly continuous dd's we need only to replace the condition $\& 2 t_{n}+\delta_{n}<a$ in Lemma 3.2 by its equivalent in terms of the starter $\left(0, \gamma_{0}, \delta_{0}\right)^{n}$ provided by Proposition 4.1.

Theorem 4.3. Let the selected $d d\left[x_{1}, x_{2} \mid \mathbf{f}\right]$ of $\mathbf{f}$ be $\omega$-regularly continuous on $D$. If the starters $x_{0}, \mathbf{A}_{0}, \gamma_{0}, \delta_{0}$ are such that

$$
\left\|x_{0}-x_{-1}\right\| \leq \gamma_{0} \quad \& \quad\left\|\mathbf{A}_{0} \mathbf{f}\left(x_{0}\right)\right\| \leq \delta_{0} \leq f_{\infty}\left(0, \gamma_{0}\right),
$$

where $f_{\infty}$ is the function of Proposition 4.1, then

(1) $\gamma_{\infty}=\delta_{\infty}=0$ and

$\underset{n}{\&}\left(\left\|x_{n}-x_{0}\right\| \leq t_{n} \leq 0.5\left(a-\delta_{n}\right) \quad \& \quad\left\|x_{n}-x_{n-1}\right\| \leq \gamma_{n} \quad \& \quad\left\|x_{n+1}-x_{n}\right\| \leq \delta_{n}\right)$;

(2) the sequence $\left(x_{n}, \mathbf{A}_{n}\right)$ generated by the method (1.5) from the starter $\left(x_{0}, \mathbf{A}_{0}\right)$ converges to a solution $\left(x_{\infty}, \mathbf{A}_{\infty}\right)$ of the system (4.1);

(3) $x_{\infty}$ is the only solution of the equation $\mathbf{f}(x)=0$ in the ball $B\left(x_{0}, a-t_{\infty}\right)$;

(4) for all $n=0,1, \ldots$,

$$
\begin{aligned}
& \left\|\mathbf{f}\left(x_{n+1}\right)\right\| \leq \delta_{n}\left(\omega\left(a-2 t_{n}+\gamma_{n}\right)-\omega\left(a-2 t_{n}-\delta_{n}\right)\right), \\
& \Delta_{n}:=\left\|x_{\infty}-x_{n}\right\| \leq t_{\infty}-t_{n}, \\
& \frac{\Delta_{n+1}}{\Delta_{n}} \leq \frac{\omega\left(\Delta_{n-1}\right)}{\omega\left(a-2 t_{n}+\gamma_{n}\right)} .
\end{aligned}
$$

For operators with Lipschitz continuous dd's, we use in Lemma 3.2 the equivalent of the condition $\underset{n}{\&} 2 t_{n}+\delta_{n}<a$ provided by Proposition 4.2:

$$
\underset{n}{\&} 2 t_{n}+\delta_{n}<c^{-1}-\gamma_{0} \Longleftrightarrow 4 c^{-1} \delta_{0} \leq\left(c^{-1}-\gamma_{0}\right)^{2} .
$$

The result is the following:

Corollary 4.4. Let the selected $d d\left[x_{1}, x_{2} \mid \mathbf{f}\right]$ of $\mathbf{f}$ be Lipschitz continuous on $D$ in the sense of (2.2). If the starters $x_{0}, x_{-1}, \mathbf{A}_{0}, \gamma_{0}, \delta_{0}$ are such that

then

$$
\left\|x_{0}-x_{-1}\right\| \leq \gamma_{0} \quad \& \quad\left\|\mathbf{A}_{0} \mathbf{f}\left(x_{0}\right)\right\| \leq \delta_{0} \leq \frac{a^{2}}{4\left(a+\gamma_{0}\right)},
$$

(1) $\gamma_{\infty}=\delta_{\infty}=0, I_{0}:=\left(c^{-1}-\gamma_{0}\right)^{2}-4 c^{-1} \delta_{0} \geq 0$, and for all $n \geq 1$

$$
\begin{aligned}
\left\|x_{n}-x_{0}\right\| & \leq t_{n}=0.5\left(c^{-1}-\gamma_{0}-\delta_{n}-\sqrt{\delta_{n}^{2}+\gamma_{n} \delta_{n}+0.25 I_{0}}\right), \\
\left\|x_{n}-x_{n-1}\right\| & \leq \gamma_{n},
\end{aligned}
$$




$$
\left\|\mathbf{A}_{n} \mathbf{f}\left(x_{n}\right)\right\| \leq \delta_{n}
$$

(2) the sequence $\left(x_{n}, \mathbf{A}_{n}\right)$ generated by the method (1.5) from the starter $\left(x_{0}, \mathbf{A}_{0}\right)$ remains in the ball $B\left(\left(x_{0}, \mathbf{A}_{0}\right),\left(t_{\infty}, r_{\mathbf{A}}\right)\right)$, where

$$
t_{\infty}=0.5\left(c^{-1}-\gamma_{0}-\sqrt{I_{0}}\right), r_{\mathrm{A}}:=\frac{c^{-1}-\gamma_{0}-\delta_{0}-\sqrt{I_{0}}}{c\left(c^{-1}-\gamma_{0}-\delta_{0}\right) \sqrt{I_{0}}}+\frac{\gamma_{0}+\delta_{0}}{1-c\left(\gamma_{0}+\delta_{0}\right)},
$$

and converges to a solution $\left(x_{\infty}, \mathbf{A}_{\infty}\right)$ of the system

$$
\mathbf{f}(x)=0 \quad \& \quad \mathbf{A}[x, x \mid \mathbf{f}]=\mathbf{I}
$$

(3) $x_{\infty}$ is the only solution of the equation $\mathbf{f}(x)=0$ in the ball

$$
B\left(x_{0}, 0.5\left(a-t_{\infty}\right)\right) \text {; }
$$

(4) for all $n=0,1, \ldots$,

$$
\begin{aligned}
& \left\|\mathbf{f}\left(x_{n+1}\right)\right\| \leq c \delta_{n}\left(\gamma_{n}+\delta_{n}\right), \\
& \Delta_{n}:=\left\|x_{\infty}-x_{n}\right\| \leq \delta_{n}+\sqrt{\delta_{n}^{2}+\gamma_{n} \delta_{n}+0.25 I_{0}}-0.5 \sqrt{I_{0}}, \\
& \frac{\Delta_{n+1}}{\Delta_{n}} \leq \frac{\Delta_{n-1}}{\gamma_{n}+2 \sqrt{\gamma_{n}^{2}+\gamma_{n} \gamma_{n-1}+0.25 I_{0}}} .
\end{aligned}
$$

\section{Rate of convergence}

The bound (4.7) shows that the sequence $x_{n}$ converges (if and when) to $x_{\infty}$ superlinearly. It should be stressed that this result applies also to nonsmooth operators. Superlinear convergence alone, however, is insufficient to judge merits of an iterative method. Theorem 4.3 offers a more firm ground for comparison of various methods. By (4.7),

$$
\Delta_{n+1} \leq \Delta_{n} \omega\left(\Delta_{n-1}\right) / \omega\left(a-2 t_{\infty}\right)=\Delta_{n} \omega_{0}\left(\Delta_{n-1}\right),
$$

where $\omega_{0}(t):=\omega(t) / \omega\left(a-2 t_{\infty}\right)$. Consider the related system of difference equations:

$$
u_{+}:=u \omega_{0}(v), v_{+}:=u .
$$

It is easy to see that this generator produces a majorant sequence $u_{n}$ for $\Delta_{n}$ : $\Delta_{0} \leq u_{0} \Longrightarrow \underset{n}{\&} \Delta_{n} \leq u_{n}$. This sequence can be described by a one-dimensional difference equation of the type $u_{n+1}=f\left(u_{n}\right)$. If such $f$ exists, then by (5.1) $v=f(u)$ and $v_{+}=u=f\left(u_{+}\right)=f\left(u \omega_{0}(v)\right)=f\left(u \omega_{0}(f(u))\right)$, so that $f$ must satisfy the functional equation

$$
x\left(u \omega_{0}(x(u))\right)=u .
$$

Conversely, a solution $f$ of this equation determines the one-dimensional difference equation $u_{n+1}=f\left(u_{n}\right)$ that, given $u_{0}$, generates the sequence $u_{n}$ of estimates for errors $\Delta_{n}: \underset{n}{\&} \Delta_{n} \leq u_{n}$. By Theorem 4.3, $\Delta_{0} \leq t_{\infty}$, so that $t_{\infty}$ is a natural candidate for $u_{0}$. Thus, if one knows a solution of the functional equation (5.2), he is able to get a priori estimations for $\Delta_{n}$ before running the 
infinite-dimensional process (1.5). The equation (5.2) can be solved numerically by one of the iterative methods applicable to nondifferentiable operators, Broyden's method included.

The above remark suggests a novel approach to comparison of efficiency of iterative methods. Consider a situation when analyses of two iterative methods

$$
x_{+}:=\mathbf{F}_{i}(x), i=1,2,
$$

produced two sequences $u_{n}^{(1)}$ and $u_{n}^{(2)}$ of upper bounds for the respective errors $\Delta_{n}^{(1)}$ and $\Delta_{n}^{(2)}$. Clearly, if $\underset{n}{\&} u_{n}^{(1)} \leq u_{n}^{(2)}$, we would prefer the first method. However, it is an ideal situation. More realistically, suppose that ${\underset{n=0}{\&}}_{n}^{m} u_{n}^{(1)} \leq u_{n}^{(2)}$ and $\stackrel{\infty}{n=m+1}_{m}^{\infty} u_{n}^{(2)} \leq u_{n}^{(1)}$. Then, the obvious decision is to use $\mathbf{F}_{1}$ for the first

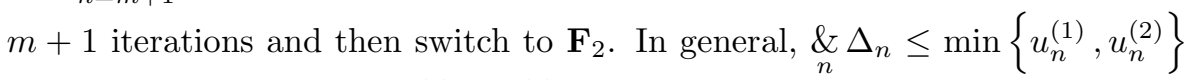
and one should use $\mathbf{F}_{1}$, if $u_{n}^{(1)} \leq u_{n}^{(2)}$, and $\mathbf{F}_{2}$, otherwise. We see that different methods can be combined to create new more efficient ones.

In the case of linear $\omega,(4.8)$ implies

$$
\Delta_{n+1} \leq \alpha \Delta_{n} \Delta_{n-1}, \alpha:=I_{0}^{-1 / 2}
$$

or, equivalently, $\alpha \Delta_{n+1} \leq \alpha \Delta_{n} \cdot \alpha \Delta_{n-1}$. The corresponding difference equation is $u_{n+1}=u_{n} u_{n-1}$. The variable change $v_{n}=\ln u_{n}$ yields $v_{n+1}=v_{n}+v_{n-1}$, the familiar Fibonacci difference equation [1]. Its solution is

$$
v_{n}=F i b_{n-1} v_{1}+F i b_{n} v_{0},
$$

where $F i b_{n}$ is the $n$-th Fibonacci number:

$$
F i b_{n}=\frac{1}{\sqrt{5}}\left(\frac{1+\sqrt{5}}{2}\right)^{n}-\frac{1}{\sqrt{5}}\left(\frac{1-\sqrt{5}}{2}\right)^{n} \text {. }
$$

It follows that

$$
\begin{aligned}
\alpha \Delta_{n} & \leq u_{n}=\exp \left(v_{n}\right)=\exp \left(F i b_{n-1} v_{1}+F i b_{n} v_{0}\right) \\
& =\exp \left(F i b_{n-1} \ln u_{1}+F i b_{n} \ln u_{0}\right) \\
& =\exp \left(\ln \left(u_{1}^{F i b_{n-1}} u_{0}^{F i b_{n}}\right)\right)=u_{1}^{F i b_{n-1}} u_{0}^{F i b_{n}} .
\end{aligned}
$$

By Corollary $4.4, \Delta_{0} \leq 0.5\left(a-\sqrt{I_{0}}\right)$ and

$$
\Delta_{1} \leq \delta_{1}+\sqrt{\delta_{1}^{2}+\delta_{1} \delta_{0}+0.25 I_{0}}-0.5 \sqrt{I_{0}} .
$$

So, we can take $u_{0}=0.5\left(a-\sqrt{I_{0}}\right)$ and

$$
u_{1}=\delta_{1}+\sqrt{\delta_{1}^{2}+\delta_{1} \delta_{0}+0.25 I_{0}}-0.5 \sqrt{I_{0}},
$$

where $\delta_{1}=\delta_{0}\left(\gamma_{0}+\delta_{0}\right) /\left(a-\delta_{0}\right)$ by (4.5). Hence,

$$
\Delta_{n} \leq \sqrt{I_{0}} u_{1}^{F i b_{n-1}} u_{0}^{F i b_{n}}, u_{0}:=0.5\left(a-\sqrt{I_{0}}\right),
$$




$$
u_{1}:=\delta_{1}+\sqrt{\delta_{1}^{2}+\delta_{1} \delta_{0}+0.25 I_{0}}-0.5 \sqrt{I_{0}}
$$

\section{Applications}

As seen from (1.5), implementation of the method does not require the knowledge of the regularity modulus or the Lipschitz constant, which are needed for convergence analysis. One should also has in mind that the convergence conditions given by Theorem 4.3 and Corollary 4.4 are only sufficient and so the iterations (1.5) may converge even when these conditions are violated. Therefore, the lack of information about properties of the operator of interest should not prevent a practitioner from using the method. The applications presented in this section can serve as illustrations for this remark.

\subsection{Complementarity problem}

Let $\mathbb{H}, C$, and $\mathbf{g}: \mathbb{H} \rightarrow \mathbb{H}$ be a Hilbert space, a closed cone in it, and a (generally nonsmooth) operator acting on $\mathbb{H}$, respectively. The problem of finding a solution of the system

$$
x \in C \quad \& \mathbf{g}(x) \in C^{*} \&\langle x, \mathbf{g}(x)\rangle=0,
$$

where $C^{*}$ is the dual cone: $C^{*}:=\{y \in \mathbb{H} \mid\langle x, y\rangle \geq 0, \forall x \in C\}$, is called [9] complementarity problem (briefly $\mathrm{CP}(C, \mathbf{g})$ ). Its special case

$$
x \in \mathbb{E}^{n} \quad \& \quad \&_{i=1}^{n}\left(x_{i} \geq 0 \quad \& \quad g_{i}(x) \geq 0 \quad \& \quad x_{i} g_{i}(x)=0\right)
$$

is the subject of vast literature (see [4],[9]). In particular, Mangasarian in [18] proved a theorem establishing equivalence between the problem (6.2) and an operator equation. His result can be stated as:

Proposition 6.1 ([18]). Let $C$ and $\varphi: \mathbb{R} \rightarrow \mathbb{R}$ be the standard positive cone of $\mathbb{E}^{n}$ and any strictly increasing function with $\varphi(0)=0$. Define the operator $\mathbf{f}: \mathbb{E}^{n} \rightarrow \mathbb{E}^{n}$ by setting

$$
\mathbf{f}(x)_{i}:=\varphi\left(\left|x_{i}-g_{i}(x)\right|\right)-\varphi\left(x_{i}\right)-\varphi\left(g_{i}(x)\right), i=1, \ldots, n .
$$

$A$ vector $x=\left(x_{1}, \ldots, x_{n}\right)$ solves $C P(C, \mathbf{g})$ if and only if $\mathbf{f}(x)=0$.

Mangasarian's theorem can be extended to any separable Hilbert space $\mathbb{H}$ using the fact that it has an orthonormal basis $\left\{e_{k}\right\}_{1}^{\infty}$ (see, for example, [11, Ch.IV] or [3, Corollary 2.1.8]), so that each $x \in \mathbb{H}$ has the unique representation $x=\sum_{1}^{\infty}\left\langle x, e_{k}\right\rangle e_{k}$. Taking for $C$ the standard positive cone

$$
C:=\left\{x \in \mathbb{H} \mid \underset{k}{\&}\left\langle x, e_{k}\right\rangle \geq 0\right\},
$$

we obtain $C^{*}=C$ and $\mathbf{g}(x) \in C^{*} \Longleftrightarrow \underset{k}{\&}\left\langle\mathbf{g}(x), e_{k}\right\rangle \geq 0$. With this choice of $C, \operatorname{CP}(C, \mathbf{g})$ becomes: find $x \in \mathbb{H}$ such that

$$
\&_{k}\left(\left\langle x, e_{k}\right\rangle \geq 0 \quad \& \quad\left\langle\mathbf{g}(x), e_{k}\right\rangle \geq 0 \quad \& \quad\left\langle x, e_{k}\right\rangle\left\langle\mathbf{g}(x), e_{k}\right\rangle=0\right) .
$$


Proposition 6.2. Let $\mathbb{H}$ and $\left\{e_{k}\right\}_{1}^{\infty}$ be a separable Hilbert space and an orthonormal basis in it, respectively. Let $\varphi$ be any strictly increasing function on $\mathbb{R}$ with $\varphi(0)=0$. Define $\mathbf{f}: \mathbb{H} \rightarrow \mathbb{H}$ by setting coordinates $\left\langle\mathbf{f}(x), e_{k}\right\rangle$ of $\mathbf{f}(x)$ to

$$
\varphi\left(\left|\left\langle\mathbf{g}(x)-x, e_{k}\right\rangle\right|\right)-\varphi\left(\left\langle\mathbf{g}(x), e_{k}\right\rangle\right)-\varphi\left(\left\langle x, e_{k}\right\rangle\right) .
$$

Then $x \in \mathbb{H}$ solves (6.3) if and only if $\mathbf{f}(x)=0$.

Proof. Fix a $k \in \mathbb{N}$ and let for short $a:=\left\langle x, e_{k}\right\rangle$ and $b:=\left\langle\mathbf{g}(x), e_{k}\right\rangle$. The claim reduces to

$$
a \geq 0 \quad \& \quad b \geq 0 \quad \& \quad a b=0 \Longleftrightarrow \varphi(|a-b|)=\varphi(a)+\varphi(b) .
$$

Because of the symmetry $a \leftrightarrow b$, it suffices to consider the case $a \geq b$. If the left side of (6.4) is true, then $a \geq b=0$ and so the right side is true also. Conversely, suppose that $\varphi(a-b)=\varphi(a)+\varphi(b)$. The following situations are conceivable:

(i) $a \geq b>0$, (ii) $a \geq b=0$, (iii) $a>0>b$, (iv) $a=0>b$, (v) $0>a \geq b$.

We must show that only (ii) is possible if $\varphi(a-b)=\varphi(a)+\varphi(b)$. Indeed, as $\varphi$ is increasing and $\varphi(0)=0$,

$$
\begin{gathered}
(\mathrm{i}) \Longrightarrow \varphi(a) \geq \varphi(b)>0 \Longrightarrow \varphi(a)>\varphi(a-b)=\varphi(a)+\varphi(b)>\varphi(a), \\
(\text { iii }) \Longrightarrow \varphi(a)>0>\varphi(b) \Longrightarrow \varphi(a)<\varphi(a-b)=\varphi(a)+\varphi(b)<\varphi(a), \\
\begin{aligned}
(\mathrm{iv}) \Longrightarrow \varphi(a)=0>\varphi(b) \Longrightarrow 0 & =\varphi(a)<\varphi(|b|)=\varphi(a-b)=\varphi(a)+\varphi(b) \\
& =\varphi(b)<0, \\
(\mathrm{v}) \Longrightarrow 0>\varphi(a) \geq \varphi(b) \Longrightarrow 0 & \leq \varphi(a-b)=\varphi(a)+\varphi(b)<0 .
\end{aligned}
\end{gathered}
$$

Thus, in each of these four cases, $\varphi(a-b) \neq \varphi(a)+\varphi(b)$, while (ii) implies $a \geq 0 \quad \& \quad b \geq 0 \quad \& \quad a b=0$.

Inasmuch as the Hilbert space $L_{2}[0,1]$ of square integrable functions on $[0,1]$ is separable [11], [3] (and so has an orthonormal basis) and because the function $t \mapsto t$ can be taken for $\varphi$, we have:

Corollary 6.3. A function $x \in L_{2}[0,1]$ solves the system

$$
\&_{0 \leq t \leq 1}(x(t) \geq 0 \quad \& \quad \mathbf{g}(x)(t) \geq 0 \quad \& \quad x(t) \mathbf{g}(x)(t)=0)
$$

if and only if $\mathbf{f}(x)(t):=\min \{x(t), \mathbf{g}(x)(t)\}=0$.

We have applied the method (1.5) to the problem (6.5) with

$$
\mathbf{g}(x)(t):=x(t)-(t-c)(2-t),
$$

where $c$ is some constant within $(0,1)$. For this $\mathbf{g}$, the problem $(6.5)$ has the only solution $x(t)=(t-c)^{+}(2-t)$. The successive approximations $x_{n}$ were represented in computer memory by the vector of their values at the knots of the 


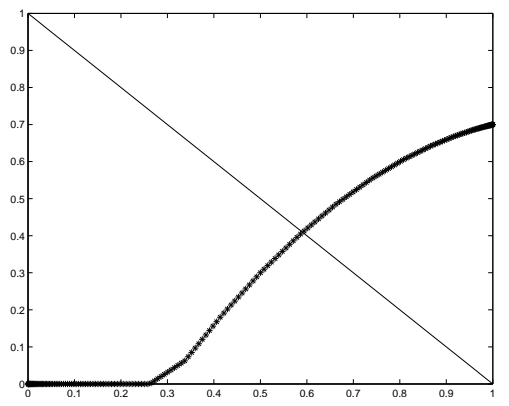

Figure 1. Complementarity problem

Tchebyshev mesh of 21 points on $[0,1]$. Starting from $x_{0}(t):=1-t, x_{-1}(t):=$ $0.9 x_{0}(t)+0.001$, and

$$
\left(\mathbf{A}_{0} h\right)(t):=\frac{x_{0}(t)-x_{-1}(t)}{\mathbf{f}\left(x_{0}\right)(t)-\mathbf{f}\left(x_{-1}\right)(t)} h(t),
$$

the method attains the requested accuracy of approximation (measured by the max-norm of the function $\mathbf{f}(x)(t))$ of $10^{-12}$ after 8 iterations. The next table shows the errors $\left\|\mathbf{f}\left(x_{n}\right)(t)\right\|$ versus the iteration number $n$ for $c=0.3$. The figure captioned "Complementarity problem" shows the initial (the solid line) and the final (the asterisked line) approximations to the solution.

\begin{tabular}{|c|l|}
\hline$n$ & Error \\
\hline 0 & $1.00 e 0$ \\
1 & $7.00 e-1$ \\
2 & $7.00 e-1$ \\
3 & $1.37 e 0$ \\
4 & $7.62 e-2$ \\
5 & $7.63 e-2$ \\
6 & $4.80 e-12$ \\
7 & $1.28 e-12$ \\
8 & $7.32 e-13$ \\
\hline
\end{tabular}

\subsection{A functional equation}

We apply here Broyden's method to the functional equation (5.2) with $\omega_{0}(t):=\sqrt{t}$. With $x_{0}(t):=t, x_{-1}(t):=0.9 x_{0}(t)$, and $\mathbf{A}_{0}$ as in (6.6), the max-norm of the function $\mathbf{f}(x)(t):=x\left(t \omega_{0}(x(t))-t\right.$ has been reduced to $10^{-12}$ after 40 iterations. The figure captioned "Functional equation" shows the initial (the solid line) and the final (the dashed line) approximations to the solution. 


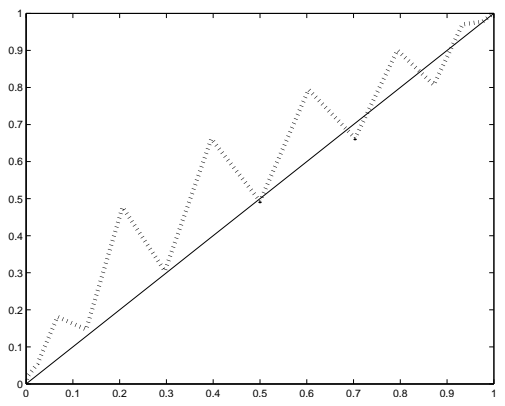

Figure 2. Functional equation

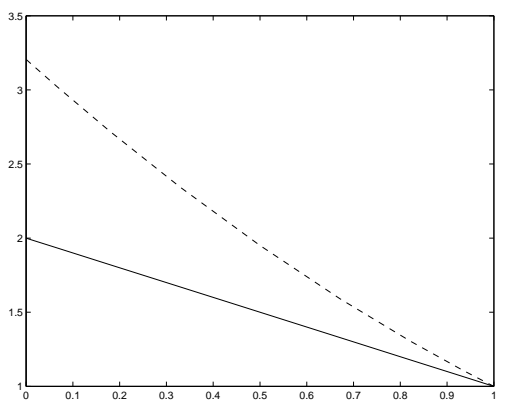

Figure 3. Integral equation

\subsection{An integral equation}

Pimbley [19] considered the following integral equation arising in a model of statistical mechanics:

(6.7) $\mathbf{f}(x)(t):=x(t)-\lambda \int_{t}^{1} x(s-t) x(s) d s-1=0, \quad 0 \leq t \leq 1, \quad x \in \mathbb{C}[0,1]$.

He has found that it has two positive decreasing twice differentiable solutions for each $\lambda \in(0,0.5)$, one for $\lambda=0.5$, and none for $\lambda>0.5$, and has investigated their properties. However, he did not try to solve this equation numerically.

We apply the method (1.5) to the equation (6.7) with $\lambda=0.5$ starting from $x_{0}(t):=x_{-1}(t):=2-t$ and $\mathbf{A}_{0}:=\mathbf{I}$. The successive approximations $x_{n}(t)$ were represented by cubic splines created on the Tchebyshev mesh of 16 points on $[0,1]$. The quality of an approximation $x_{n}$ is measured by the max-norm of the corresponding function (6.7). It is shown in the table below under the caption Error. The final approximation $x_{15}$ is tabulated in columns 3 and 4 of the table. The figure captioned "Integral equation" shows the initial (the solid line) and the final (the dashed line) approximations. 


\begin{tabular}{|c|l|c|c|}
\hline Iteration & Error & $t$ & $x_{15}(t)$ \\
\hline 0 & $1.6597 \mathrm{e}-1$ & $0.0000 \mathrm{e}-0$ & $3.0410 \mathrm{e}+0$ \\
1 & $1.1076 \mathrm{e}-1$ & $3.1439 \mathrm{e}-3$ & $3.0329 \mathrm{e}+0$ \\
2 & $4.6124 \mathrm{e}-2$ & $2.8058 \mathrm{e}-2$ & $2.9698 \mathrm{e}+0$ \\
3 & $2.2092 \mathrm{e}-2$ & $7.6638 \mathrm{e}-2$ & $2.8485 \mathrm{e}+0$ \\
4 & $7.2765 \mathrm{e}-3$ & $1.4645 \mathrm{e}-1$ & $2.6781 \mathrm{e}+0$ \\
5 & $9.3770 \mathrm{e}-4$ & $2.3398 \mathrm{e}-1$ & $2.4714 \mathrm{e}+0$ \\
6 & $6.1311 \mathrm{e}-5$ & $3.3486 \mathrm{e}-1$ & $2.2428 \mathrm{e}+0$ \\
7 & $6.6470 \mathrm{e}-6$ & $4.4402 \mathrm{e}-1$ & $2.0071 \mathrm{e}+0$ \\
8 & $4.8785 \mathrm{e}-6$ & $5.5598 \mathrm{e}-1$ & $1.7782 \mathrm{e}+0$ \\
9 & $3.3993 \mathrm{e}-6$ & $6.6514 \mathrm{e}-1$ & $1.5677 \mathrm{e}+0$ \\
10 & $3.3742 \mathrm{e}-7$ & $7.6602 \mathrm{e}-1$ & $1.3843 \mathrm{e}+0$ \\
11 & $2.3144 \mathrm{e}-9$ & $8.5355 \mathrm{e}-1$ & $1.2338 \mathrm{e}+0$ \\
12 & $1.0981 \mathrm{e}-9$ & $9.2336 \mathrm{e}-1$ & $1.1196 \mathrm{e}+0$ \\
13 & $4.7155 \mathrm{e}-10$ & $9.7194 \mathrm{e}-1$ & $1.0431 \mathrm{e}+0$ \\
14 & $4.7808 \mathrm{e}-12$ & $9.9686 \mathrm{e}-1$ & $1.0048 \mathrm{e}+0$ \\
15 & $3.8636 \mathrm{e}-14$ & $1.0000 \mathrm{e}+0$ & $1.0000 \mathrm{e}+0$ \\
\hline
\end{tabular}

\section{References}

[1] M. Aigner, Discrete Mathematics, American Mathematical Society, Providence, RI, 2007.

[2] C. G. Broyden, A class of methods for solving nonlinear simultaneous equations, Math. Comp. 19 (1965), 577-593.

[3] Y. Eidelman, V. Milman, and A. Tsolomitis, Functional Analysis, American Mathematical Society, Providence, RI, 2004.

[4] F. Facchinei and J.-S. Pang, Finite-dimensional Variational Inequalities and Complemetarity Problems, Springrer-Verlag, N.Y., 2003.

[5] A. Galperin, Secant method with regularly continuous divided differences, J. Comput. Appl. Math. 193 (2006), no. 2, 574-595.

[6] - On a class of systems of difference equations and their invariants, J. Difference Equ. Appl. 13 (2007), no. 5, 357-381.

[7] ${ }_{2}$, Ulm's method without derivatives, Nonlinear Anal. 71 (2009), no. 5-6, 20942113.

[8] A. Griewank, The local convergence of Broyden-like methods on Lipschitzian problems in Hilbert space, SIAM J. Numer. Anal. 24 (1987), no. 3, 684-705.

[9] P. T. Harker and J.-S. Pang, Finite-dimensional variational inequality and nonlinear complementarity problems: a survey of theory, algorithms and applications, Math. Program. 48 (1990), no. 2, 161-220.

[10] D. M. Hwang and C. T. Kelley, Convergence of Broyden's method in Banach spaces, SIAM J. Optim. 2 (1992), no. 3, 505-532.

[11] L. V. Kantorovich and G. P. Akilov, Functional Analysis, Pergamon Press, Elmsford, 1982.

[12] C. T. Kelley, Iterative methods for linear and nonlinear equations, SIAM, Philadelphia, 1995.

[13] C. T. Kelley and E. W. Sachs, Broyden's method for approximate solution of nonlinear integral equation, J. Integral Equations Appl. 9 (1985), no. 1, 25-43. 
[14] _ A quasi-Newton method for elliptic boundary value problems, SIAM J. Numer. Anal. 24 (1987), no. 3, 516-531.

[15] _ A pointwise quasi-Newton method for unconstrained optimal control problems, Numer. Math. 55 (1989), no. 2, 159-176.

[16] _ A new proof of superlinear convergence for Broyden's method in Hilbert space, SIAM J. Optim. 1 (1991), no. 1, 146-150.

[17] G. G. Magaril-Il'yaev and V. M. Tikhomirov, Convex Analysis, Theory and Applications, American Mathematical Society, Providence, RI, 2003.

[18] O. Mangasarian, Equivalence of the complementarity problem to a system of nonlinear equations, SIAM J. Appl. Math. 31 (1976), no. 1, 89-92.

[19] G. Pimbley, Positive solutions of a quadratic integral equation, Arch. Ration. Mech. Anal. 24 (1967), 107-127.

[20] L. Qi, On superlinear convergence of quasi-Newton methods for nonsmooth equations, Oper. Res. Lett. 20 (1997), no. 5, 223-228.

[21] E. W. Sachs, Broyden's method in Hilbert space, Math. Program. 35 (1986), no. 1, 71-82.

[22] W.-H. Yu, A quasi-Newton method in infinite-dimensional spaces and its application for solving a parabolic inverse problem, J. Comput. Math. 16 (1998), no. 4, 305-318.

Department of Mathematics

Ben-Gurion University of the Negev

Beer-Sheva 84105, IsRael

E-mail address: gal@math.bgu.ac.il, galperin3@gmail.com 